\title{
La imagen personal frente a los nuevos desafíos 2020 Proyecto de Investigación \\ $\mathbf{N}^{\circ} 17.4$
}

Equipo de Investigación ${ }^{(1)}$

María Pia Estebecorena

por Facultad de Diseño y Comunicación, Universidad de Palermo (ARG) y Asociación Internacional de Asesores de Imagen AICI (ARG)

\begin{abstract}
Resumen: El Proyecto de Investigación 17.4 presenta cómo los cambios sucedidos a escala global en la economía y la "nueva normalidad" como forma de vida diaria, hacen que el concepto de Imagen esté cambiando hacia el encuentro de una nueva definición que permita dar respuesta a las nuevas variables con las que se enfrenta cada persona. La percepción del tiempo, la distancia sin distancias, la virtualidad de la imagen define una nueva forma de significar el concepto Imagen. Sin duda esto afecta también no sólo lo perceptible sino la forma de estructurar la enseñanza de aspectos tales como el Color, cuando se creía que era imposible enseñarlo o diagnosticarlo virtualmente con la misma calidad con la que se aplican las técnicas en forma presencial. Todas estas variables conforman un nuevo paradigma que marcan los nuevos desafíos que se tienen al 2020 como punto de partida para repensar la Imagen dentro de este contexto con final abierto.
\end{abstract}

Palabras clave: Imagen $360^{\circ}$ - Nueva normalidad - Pandemia - Imagen Política - Psicología de la Imagen - Marca Personal - Millenialls - Habilidades Blandas - Concepto de Imagen integral - Marketing - Negocios - Sustentabilidad.

[Resúmenes en inglés y portugués en la página 275]

${ }^{(1)}$ Los CVs del Equipo de Investigación pueden consultarse en el Capítulo Directores de Líneas y Coordinadores de Proyectos de esta misma Edición.

\section{Acerca del Proyecto 17.4}

Diseño Social y Desarrollo: Percepciones sobre el Diseño Latino

Despliegue y potencial del diseño en la actualidad

El Proyecto 17.4 La Imagen personal frente a los nuevos desafíos 2020 reúne e indaga en las ideas y postulados que hacen que la Imagen constituya una representación que a partir 
de los cambios sucedidos a escala global en la economía y la "nueva normalidad" como forma de vida diaria, van conduciendo al concepto de Imagen hacia el encuentro de una nueva definición.

Se acordó entre la Universidad de Palermo y la Asociación Internacional de Asesores de Imagen (AICI, Argentina), avanzar en una investigación en la que participan académicos de ambas Instituciones, con la coordinación de María Pía Estebecorena, con el fin de generar un corpus sólido de conocimientos sobre los diversos enfoques y perspectivas relativos a los desafíos que enfrenta la imagen en relación con el contexto de la "nueva normalidad". Las reflexiones y los resultados obtenidos en el Proyecto 17.4 La imagen personal frente a los nuevos desafíos, son continuación del Proyecto 17.2 Comunicación e imagen personal $360^{\circ}$ : La imagen como concepto universal, y guarda relación con el 17.1 Cine, Moda, Cuerpo, Arte y Diseño y el 17.3 La Moda en su Laberinto en el marco de la misma Línea de Investigación y bajo la misma Directora Patricia Doria.

Sus principales objetivos son:

- Analizar las nuevas variables que han surgido producto de los cambios globales en la economía, y el concepto de "nueva normalidad", en relación con el dimensionamiento de la imagen personal social y en los negocios.

- Problematizar y teorizar acerca de los discursos de la imagen en tanto la construcción del tiempo y su percepción, la distancias de la virtualidad y los aspectos que denotan y connotan un posible cambio de paradigma.

- Estudiar como los elementos contextuales que conforman la situación del bloque regional y global reorientan este nuevo panorama para repensar las características del nuevo concepto de imagen que se abre y se proyecta.

\section{Acerca de la Línea 17}

Imagen, Moda y Tendencias dirigida por Patricia Doria se desarrolla de manera ininterrumpida desde 2015 en la Facultad de Diseño y Comunicación (UP, Argentina), e incluye hasta el momento cuatro proyectos finalizados el 17.1 Cine, Moda, Cuerpo, Arte y Diseño coordinado por Patricia Doria (UP), Denise Trindade (Universidade Estácio de Sá, Brasil) y Beatriz Ferreira Pires (Escola de Artes, Ciências e Humanidades, Universidade de São Paulo EACH-USP, Brasil), el 17.2 Comunicación e Imagen personal $360^{\circ}$ coordinado por María Pia Estebecorena y Susy Inés Bello Knoll (UP y AICI), el 17.3 La Moda en su Laberinto coordinado por Patricia Doria (UP), y el 17.4 La Imagen personal frente a los nuevos desafíos 2020 coordinado por María Pía Estebecorena (UP y AICI).

\section{Mapa de Áreas y Proyectos}

El Proyecto 17.4 se vincula con todas las carreras de grado y posgrado correspondientes a la Facultad de Diseño y Comunicación, y está claramente enlazado a las carreras de indumentaria, moda e imagen: Comunicación de Moda, Diseño de Moda, Fotografía de 
Moda, Marketing de la Moda, Moldería \& Confección y Producción de Moda. Guarda relación con la serie de proyectos de la Línea 12. Nuevos paradigmas en la enseñanza de la moda y el diseño y en la Línea 5. Cuerpo y Vestuario.

\section{Productos y Resultados}

\section{a)- Publicaciones}

Cuaderno del Centro de Estudios de Diseño y Comunicación No118 (2020/2021) La Imagen personal frente a los nuevos desafíos 2020. Coordinación María Pía Estebecorena. Facultad de Diseño y Comunicación Año XXIV, Buenos Aires, Argentina. ISSN: 16680227. Esta publicación documenta y comunica los resultados alcanzados en el proyecto de investigación 17.4 La Imagen personal frente a los nuevos desafíos 2020, y a continuación se detallan los autores y artículos contenidos en ella:

Estebecorena, María Pía (2020/2021) Prólogo. La Imagen personal frente a los nuevos desafíos 2020 (Pp. 11 a 16)

Sevilla, Coca (2020/2021) La Imagen de la Política en Pandemia: Cuando todo Comunica (Pp. 17 a 27)

Dubey Dewan, Sonia (2020/2021) The Role of Personal Image in Personal Branding (Pp. 29 a 38)

Koeck-Eripek, Eva (2020/2021) El poder de la consultoría de imagen para grupos especiales (Pp. 39 a 52)

Estebecorena, María Pía (2020/2021) El concepto de Imagen en la nueva normalidad (Pp. 53 a 60)

Malhotra, Neha (2020/2021) Bringing your Image Consulting Practices up to industry sustainability standards (Pp. 61 a 78)

Luchesi, Fernanda (2020/2021) Os erros mais comuns cometidos pelos Consultores de Imagem na hora de aplicar um plano de Marketing no negócio (Pp. 79 a 105)

Jordan, Rachel (2020/2021) Millenials x Comportamento: o Futuro das Relações (Pp. 107 a 120)

Cheong Cheok Yin, Ana: Aida, Ainun (2020/2021) Psychology of Image (Pp. 121 a 150)

Ulrich, Luciana (2020/2021) Ferramentas para o atendimento online de coloração pessoal (Pp. 151 a 161)

\section{b)- Congresos / Coloquios / Plenarios}

V Coloquio de Investigación y Desarrollo en Diseño Latino. Universidad de Palermo, 27 de julio de 2020. XI Congreso Latinoamericano de Enseñanza del Diseño. Semana Internacional del Diseño en Palermo.

Se presentaron los resultados y avances de la Línea de Investigación Nº17: Imagen, Moda y Tendencias, en dos comisiones Proyecciones de la Moda y La Imagen Personal frente a los nuevos desafíos 2020. 
En la comisión La Imagen Personal frente a los nuevos desafíos 2020, se presentaron las reflexiones y conclusiones del proyecto 18.4 del mismo nombre (Finalizado) expusieron: Coca Sevilla, Rachel Jordan, Luciana Ulrich, Maria Pía Estebecorena, Fernanda Luchessi, Ana Cheong Cheok Yin, Ainun Aida Bahardin, Sonia Dubey Dewan, Neha Malhotra y Eva Koeck Eripek.

A continuación se detallan las ponencias presentadas en esta Comisión coordinada por la Directora del proyecto María Pia Estebecorena:

Coca Sevilla (México)

La Imagen de la Política en Pandemia: cuando todo Comunica

Rachel Jordan (Brasil)

El Comportamiento Millenial X: el futuro de las relaciones

Luciana Ulrich (Brasil)

Contraste natural de la piel: por qué es esencial entender

Maria Pía Estebecorena (Argentina)

El concepto de Imagen en la nueva normalidad

Fernanda Luchessi (Brasil)

Los errores más comunes cometidos por Consultores de Imagen al aplicar un plan de marketing en el negocio

Ana Cheong Cheok Yin y Ainun Aida Bahardin (Malasia)

La psicología de la imagen

Sonia Dubey Dewan (India)

El papel de la imagen personal en la marca personal

Neha Malhotra (India)

Llevando sus prácticas de consultoría de imagen a los estándares de sostenibilidad de la industria

Eva Koeck Eripek (Austria)

El poder de la consultoría de imagen para un grupo determinado y especial

$3^{\circ}$ Plenario de Directores de Investigación DC. 26, 27 y 29 de agosto de 2019. En este tercer plenario los Directores de las Líneas y Proyectos de Investigación presentaron a sus pares y al conjunto del Programa de Investigación de la Facultad de Diseño y Comunicación, los resultados obtenidos y/o en proceso (publicaciones y acuerdos), junto con los avances de Proyectos. La Directora de la Línea de Investigación №17 presentó a sus pares, el debate y los resultados del Proyecto 17.3 La Moda en su Laberinto y el Proyecto 17.4 La Imagen Personal frente a los nuevos desafíos 2020.

\section{c)- Formación de Posgrado e Impacto curricular}

La Directora Patricia Doria forma parte del Cuerpo Académico de la Facultad de Diseño y Comunicación e incorpora los contenidos se su investigación a sus asignaturas. 


\begin{abstract}
Research Project 17.4 presents how the changes that have occurred on a global scale in the economy and the "new normality" as a way of daily life, make the concept of Image is changing towards the meeting of a new definition that allows to respond to the new variables that each person faces. The perception of time, distance without distances, the virtuality of the image defines a new way of signifying the concept of Image. Undoubtedly, this also affects not only the perceptible but the way of structuring the teaching of aspects such as Color, when it was believed that it was impossible to teach or diagnose it virtually with the same quality with which techniques are applied in person. All these variables make up a new paradigm that marks the new challenges for 2020 as a starting point to rethink the Image within this context with an open end.
\end{abstract}

Keywords: $360^{\circ}$ Image - New normality - Pandemic - Political Image - Image Psychology - Personal Brand - Millenialls - Soft Skills - Comprehensive Image Concept - Marketing Business - Sustainability.

Resumo: O Projeto de Pesquisa 17.4 apresenta como as mudanças ocorridas em escala global na economia e a "nova normalidade" como forma de vida cotidiana, fazem com que o conceito de Imagem esteja mudando em direção ao encontro de uma nova definição que permite responder ao novo variáveis que cada pessoa enfrenta. A percepção do tempo, da distância sem distâncias, da virtualidade da imagem definem uma nova forma de significar o conceito de Imagem. Sem dúvida, isso afeta também não só o perceptível, mas também a forma de estruturar o ensino de aspectos como a Cor, quando se acreditava que era impossível ensiná-la ou diagnosticá-la virtualmente com a mesma qualidade com que as técnicas são aplicadas pessoalmente. Todas essas variáveis constituem um novo paradigma que marca os novos desafios de 2020 como ponto de partida para repensar a Imagem neste contexto aberto.

Palavras chave: Imagem $360^{\circ}$ - Nova normalidade - Pandemia - Imagem política - Psicologia da imagem - Marca pessoal - Millenialls - Soft Skills - Conceito de imagem abrangente - Marketing - Negócios - Sustentabilidade.

[Las traducciones de los resúmenes fueron realizadas a través de traductor automático] 